\title{
Desarrollo de un modelo de precipitación escorrentía semi- distribuido para la evaluación de los recursos hídricos del río Jalón
}

Development of a model of semi-distributed runoff precipitation for the evaluation of the water resources of the Jalón River.

Daniel Chuquin. ${ }^{1}$, Nelson Chuquin. ${ }^{2}$, Juan Chuquin. ${ }^{3}$, Lidia Castro. ${ }^{4} \&$ Ramiro Cepeda. ${ }^{5}$

Recibido: 29-04-2019 / Revisado: 26-05-2019 /Aceptado: 28-06-2019/ Publicado: 15-07-2019

\begin{abstract}
:
DOI: https://doi.org/10.33262/cienciadigital.v3i3.1.680
\end{abstract}

The main goal of this study is the water resources evaluation in the Jalon river basin, with $9718 \mathrm{~km} 2$, and the study of the behavior of the Jalon system subbasins, one of the most important tributaries of the Ebro River.

For this goal, a semi-distributed rainfall-runoff model has been developed, in order to evaluate the effects produced over the studio basin based on the hydrological response of each hydrographic subbasin of the system. The employed model has been HBV, conceptual semi-distributed model developed by the Meteorological and Hydrological Institute of Sweden. The tool that has been employed in the model, is RS Minerve 2.0, software that simulates rainfall-runoff processes, being based on object-oriented programming.

Finally, it is remarkable the contributions generated by the model, as correctly validated, could be really useful in upcoming jobs in hydrologic planning and management, ecological flow management, demands guarantees estimations, etc.

This contributions have been compared with natural regime SIMPA model, developed by the Center for Studies and Experimentation of Public Works (CEDEX) and used by the $\mathrm{CHE}$, in order to estimate whether they are overestimating or underestimating the

\footnotetext{
1 Escuela Superior Politécnica de Chimborazo, Chimborazo, Ecuador, daniel.chuquin@espoch.edu.ec

2 Escuela Superior Politécnica de Chimborazo, Chimborazo, Ecuador, nelson.chuquin@espoch.edu.ec

3 Escuela Superior Politécnica de Chimborazo, Chimborazo, Ecuador, juan.chuquin@espoch.edu.ec

4 Escuela Superior Politécnica de Chimborazo, Chimborazo, Ecuador, lidia.castro@espoch.edu.ec

5 Escuela Superior Politécnica de Chimborazo, Chimborazo, Ecuador, ccepeda@espoch.edu.ec
} 
contributions, trying to find possible mistakes which they may be done.

Keywords: Calibration, runoff, Jalón, modeling, Rs Minerve, sub-basin, validation.

\section{Resúmen:}

El estudio tiene como principal objetivo evaluar los recursos hídricos en la cuenca del río Jalón la cual tiene una superficie de $9718 \mathrm{~km} 2$, con esto se pretende tener un conocimiento más a detalle desde el punto de vista hidrológico del comportamiento de las subcuencas que se extienden a lo largo del sistema de explotación del Jalón, la cual constituye uno de los principales afluentes del río Ebro.

Se empleó un modelo hidrológico precipitación-escorrentía (MPE) semidistribuido con el fin de evaluar los efectos que se producen sobre las características de la cuenca en estudio en base a la respuesta hidrológica de cada subcuenca hidrográfica que componen el sistema. El modelo utilizado fue el HBV que se trata de un modelo conceptual semidistribuido desarrollado por el Instituto Meteorológico e Hidrológico de Suecia. La herramienta empleada para la modelación ha sido RS Minerve 2.0, software que simula los procesos de precipitación-escorrentía y se basa en la programación orientada a objetos.

Finalmente destacar la importancia de las aportaciones generadas por el modelo, que siendo validado resulta fundamental para posteriores trabajos en materia de gestión y planificación hidrológica, gestión de caudales ecológicos, estimación de garantías de las demandas etc. Estas aportaciones han sido comparadas con las aportaciones en régimen natural del modelo SIMPA, desarrollado por el Centro de Estudios y Experimentación de Obras de Públicas (CEDEX) y empleado por la Confederación Hidrográfica del Ebro (CHE), con el fin de estimar si ellos están sobreestimando o infravalorando las aportaciones, es decir es posible determinar los posibles errores que se pueden estar cometiendo.

Palabras claves:_Calibración, escorrentía, Jalón, modelación, Rs Minerve, subcuenca, validación.

\section{Introduccion}

El agua constituye en la actualidad un recurso no renovable indispensable para el desarrollo de la vida y a la vez una de las principales materias primas en la mayoría de los procesos productivos, por tal razón surge la necesidad de implantar mecanismos para la gestión y planificación del recurso hídrico mediante la aplicación de acciones tanto estructurales (construcción de infraestructura) como medidas no estructurales(medidas que promuevan la eficiencia de uso del agua y tecnologías que disminuyan el riesgo hídrico). Dentro de la hidrología los modelos hidrológicos constituyen una herramienta que merece especial importancia para la gestión y planificación de los recursos ambientales e hídricos. Dada la 
exigencia en cuanto a la capacidad predictiva que un modelo conlleva, hoy en día existe la necesidad de fortalecer los modelos existentes.

A lo largo de la historia la modelación hidrológica ha ido cambiando continuamente, desde el Método Racional (Mulvany et al., 1850) a modelos físicos distribuidos (Wigmosta et la., 1994; Abbott et al., 1986b; Refsgaard et al., 1995; Ewen et al., 2000; Benoit et al., 2000; Bates et al., 2000; De Roo et al, 2000; Vivoni et al., 2003).Durante el mismo período, se desarrolló el hidrograma unitario simple (Sherman, 1932), modelos de entrada y salida actualmente llamados modelos basados en datos, mismos que han evolucionado a modelos de redes neuronales artificiales (García, 2001). La elección del modelo más apropiado es una tarea difícil dado que cada modelador intenta persuadir y a la vez promover su programa desarrollado.

Los modelos hidrológicos son representaciones conceptuales y simplificadas de una parte del ciclo hidrológico, generalmente son usados para la predicción y comprensión de los procesos hidrológicos que se llevan a cabo en la naturaleza. Estos toman especial importancia cuando se evidencia la carencia de base de datos que establezcan las características de la línea base (iniciales) y determinen de esta manera los impactos a largo plazo. Los modelos a su vez son capaces de simular los procesos hidrológicos en el espacio y serie de temporal deseada (De vos et al, 2006; Taffese., 2011).

El modelo hidrológico empleado en el estudio para la evaluación de los recursos hídricos es el HBV (Bergstrom et al., 1995; Lindstrom et al., 1197)) se ha elegido este modelo debido a que no tiene gran cantidad de parámetros, satisfaciendo así el principio de parsimonia y constituyendo un modelo robusto y versátil.

El software empleado en la modelación fue RS-Minerve (García et al., 2013, Jordan et al., 2010) mismo que simula la formación y propagación de la escorrentía superficial y capaz de modelar redes hidráulicas e hidrológicas complejas de acuerdo a un esquema semidistribuido conceptual, ha sido utilizado para diferentes estudios en varios países.

\section{Importancia del problema}

El objetivo principal del presente estudio es evaluar el recurso hídrico disponible en la cuenca del río Jalón que tiene un área de $9718 \mathrm{~km}^{2}$ aproximadamente, mediante el uso de modelos de precipitación-escorrentía (MPE) teniendo como primicia el análisis y diagnóstico de la gestión del sistema. Además, una vez desarrollado el modelo se compara las series de aportaciones generadas con las aportaciones provenientes del modelo SIMPA (Simulación PrecipitaciónAportación) que es el empleado por la confederación hidrográfica del Ebro para el estudio de las aportaciones en régimen natural con el fin de establecer un juicio crítico para determinar si el organismo en cuestión sobreestima o subestima los recursos disponibles para la posterior gestión del fluido en el medio. 


\section{Metodología}

La elaboración de modelos de precipitación-escorrentía (MPE) requiere de información necesaria e indispensable para la ejecución del mismo, la cual debe caracterizarse por ser representativa de la cuenca a modelar, por tal motivo, los datos disponibles atraviesan una etapa de tratamiento y depuración para posteriormente procesarla.

En la Figura 1 se especifica el procedimiento que se ha seguido para la elaboración, calibración y validación del MPE.

Figura 1. Metodología empleada para el desarrollo del modelo

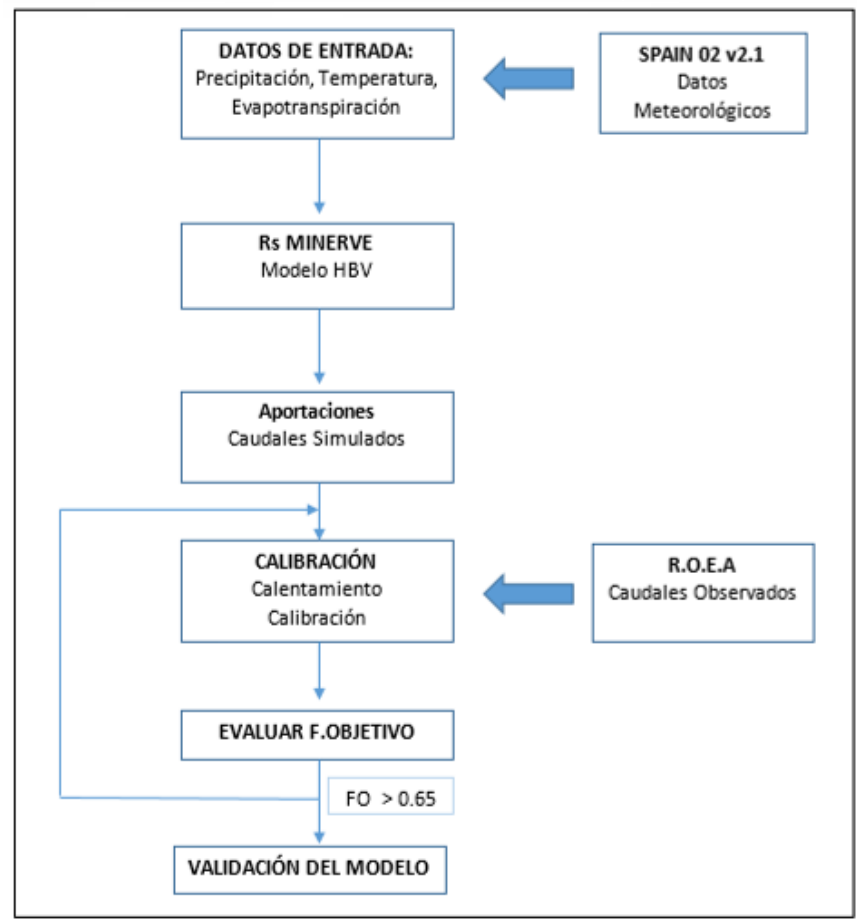

Inputs de entrada: Los datos de precipitación y temperatura se obtuvieron a partir de la base de datos del proyecto Spain 02 v2.1,[18], los datos resultan de una serie de interpolaciones empleando el método de krigeado. En cuanto a la evapotranspiración se empleó el modelo de Hargreaves (Hargreves, 1985).

$$
\text { ETo }=0.0135 * k *(T+17.78) * R a *(\operatorname{Tmax}-\operatorname{Tmin})^{0.5}
$$

Donde: Kc es el coeficiente de cultivo, ETo la evapotranspiración potencial, la Eto se calcula en función de la radiación solar incidente, del coeficiente de cultivo y de las temperaturas máximas y mínimas, mediante la Ec. 2:

$$
\text { ETo }=\left(0.0135 * k *(T+17.78) * R a *\left(\operatorname{Tmax}-T_{\operatorname{Tmin}}\right)^{0.5}\right) * 0.408
$$


Donde: ETo es la evapotranspiración potencial diaria (de referencia) $(\mathrm{mm} / \mathrm{dia}) \mathrm{T}$ la temperatura media diaria $(\mathrm{C})$, Tmax la temperatura máxima diaria $(\mathrm{C})$, Tmin la temperatura mínima diaria (C), Ra la radiación solar extraterrestre (mm/dia), K: Coeficiente de cultivo.

El coeficiente de cultivo se determinó en función de la cobertura vegetal del mismo, empleando la información que viene dado en la base de datos de usos de suelos de Corine_2006 (Coordination on information of the environmet) del instituto geográfico militar.

Aplicación del modelo HBV: El modelo HBV (Figura 2) tiene 3 principios fundamentales: la acumulación y el derretimiento de la nieve, la humedad del suelo, el ciclo fluvial de los ríos y la respuesta de los mismos respecto al proceso que se genera en la cuenca. Se basa en una modelación a través de tanques o reservorios en el cual cada uno presenta ciertas características específicas entre las que destacan el umbral para la producción de escorrentía, constantes que conceptualizan la velocidad con la que se propagan los procesos. Este modelo utiliza la precipitación, la temperatura y la evapotranspiración potencial como parámetros de entrada a partir de los cuales se estima la producción de escorrentía a través de la cuenca.

$$
Q t o t a l=Q r+Q u+Q l
$$

Donde: Qr es la escorrentía directa $\left(\mathrm{m}^{3} / \mathrm{s}\right)$, Qu es el flujo procedente del reservorio superior $\left(\mathrm{m}^{3} / \mathrm{s}\right)$ y Q1 el flujo base $\left(\mathrm{m}^{3} / \mathrm{s}\right)$.

Figura 2. Conceptualización del modelo HBV

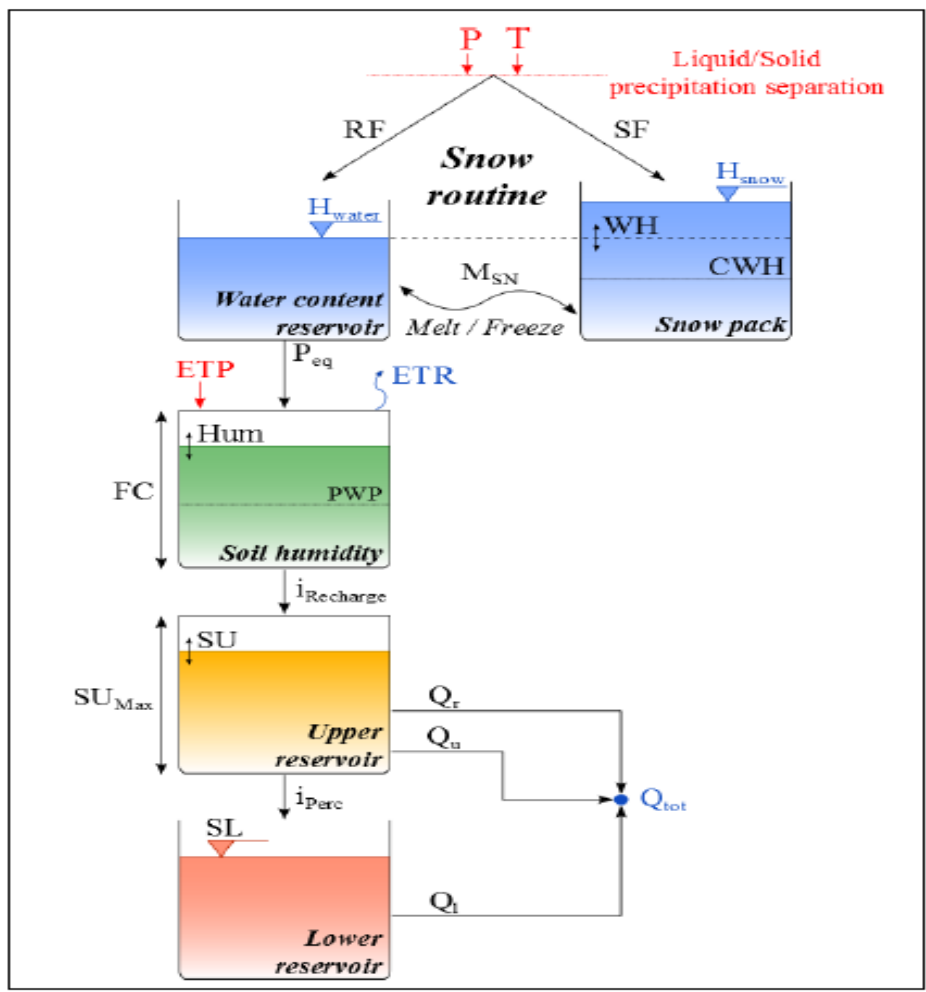


Régimen de Aportaciones, calibración y validación del modelo: Se ejecutó el modelo HBV a través del software Rs Minerve con los respectivos datos meteorológicos, generando así el régimen de aportaciones para el periodo 1987-2007 (20 años hidrológicos) para cada subcuenca del sistema de explotación del río Jalón.

Previo al periodo de calibración se realizó una fase de calentamiento ( 3 años) con el fin de establecer las condiciones iniciales del suelo para cada una de las subcuencas del sistema y minimizar así los efectos que podrían ocasionar en la etapa de calibración.

La calibración de las subcuencas (12 años) se lo realizó en función de los caudales registrados en las estaciones de aforo existentes en la cuenca (39 estaciones de la Red Oficial de Estaciones de Aforo (R.O.E.A)) que corresponden a la desembocadura de los diferentes subsistemas hídricos que componen el sistema del río Jalón.

Finalmente se realizó una etapa de validación del modelo (5 años) en donde se evaluó la funcionalidad y eficiencia del HBV.

Tanto para la calibración como validación del régimen de aportaciones (caudales) generados por el modelo HBV se empleó una función objetivo (FO) (enfatizan los diferentes aspectos y características de un hidrograma y al mismo tiempo establecen de manera aproximada el rendimiento del conjunto de parámetros seleccionado), que sea capaz de reproducir adecuadamente los caudales registrados en las estaciones de aforo, de este modo se estima la incertidumbre de las series de caudales determinados por el modelo desarrollado.

$$
\text { Fobjetivo }=F 1+F 2+F 3+F 4
$$

Donde: F1 - Índice de Nash: Estima el error cuadrático medio de la varianza normalizada observada, dando el peso más significativo a grandes errores, los cuales no necesariamente ocurren en períodos de gran cantidad de flujo.

$$
F_{1}=1-\frac{\sum_{i=1}^{n}\left(Q_{s i m, i}-Q_{i}\right)^{2}}{\sum_{i=1}^{n}\left(Q_{i}-\bar{Q}\right)^{2}}
$$

F2- Indice de Nash - in (Logarítmico): Transforma flujos que conllevan grandes pesos en errores durante flujos bajos.

$$
F_{2}=1-\frac{\sum_{i=1}^{n}\left(\ln \left(Q_{s i m, i}\right)-\ln \left(Q_{o b s, i}\right)\right)^{2}}{\sum_{i=1}^{n}\left(\ln \left(Q_{o b s, i}\right)-\ln (\bar{Q} o b s)\right)^{2}}
$$


F3- Coeficiente de correlación de Pearson: Mide la covarianza de los simulados y observados.

$$
F_{3}=1-\frac{\sum_{i=1}^{n}(Q o b s, i-\bar{Q} o b s) * \sum_{i=1}^{n}(Q \operatorname{sim}, i-\bar{Q} \operatorname{sim})}{\sqrt{\sum_{i=1}^{n}(Q o b s, j-\bar{Q} o b s)^{2}} * \sqrt{\sum_{i=1}^{n}(Q \operatorname{sim}, j-\bar{Q} \operatorname{sim})^{2}}}
$$

F4 - Bias Score: Medida simétrica entre el promedio de los valores simulados y el promedio de los de los valores observados.

$$
F_{4}=1-\left[\max \left(\frac{\bar{Q} \operatorname{sim}}{Q o b s}, \frac{\bar{Q} o b s}{\bar{Q} \operatorname{sim}}\right)-1\right]^{2}
$$

\section{Resultados}

En la Tabla 1 se presenta un resumen detallado de los periodos bajo los cuales se desarrolló la calibración y validación para cada una de las subcuencas del sistema de explotación del río Jalón (Figura 3), además se detalla los resultados de la función objetivo global en base a los cuales se establecieron los juicios críticos para determinar la validez del MPE desarrollado para obtener el régimen de aportaciones para la serie hidrológica 1985 - 2007.

Figura 3. MPE desarrollado para el sistema de explotación del río Jalón a partir del cual se obtuvieron el régimen de aportaciones

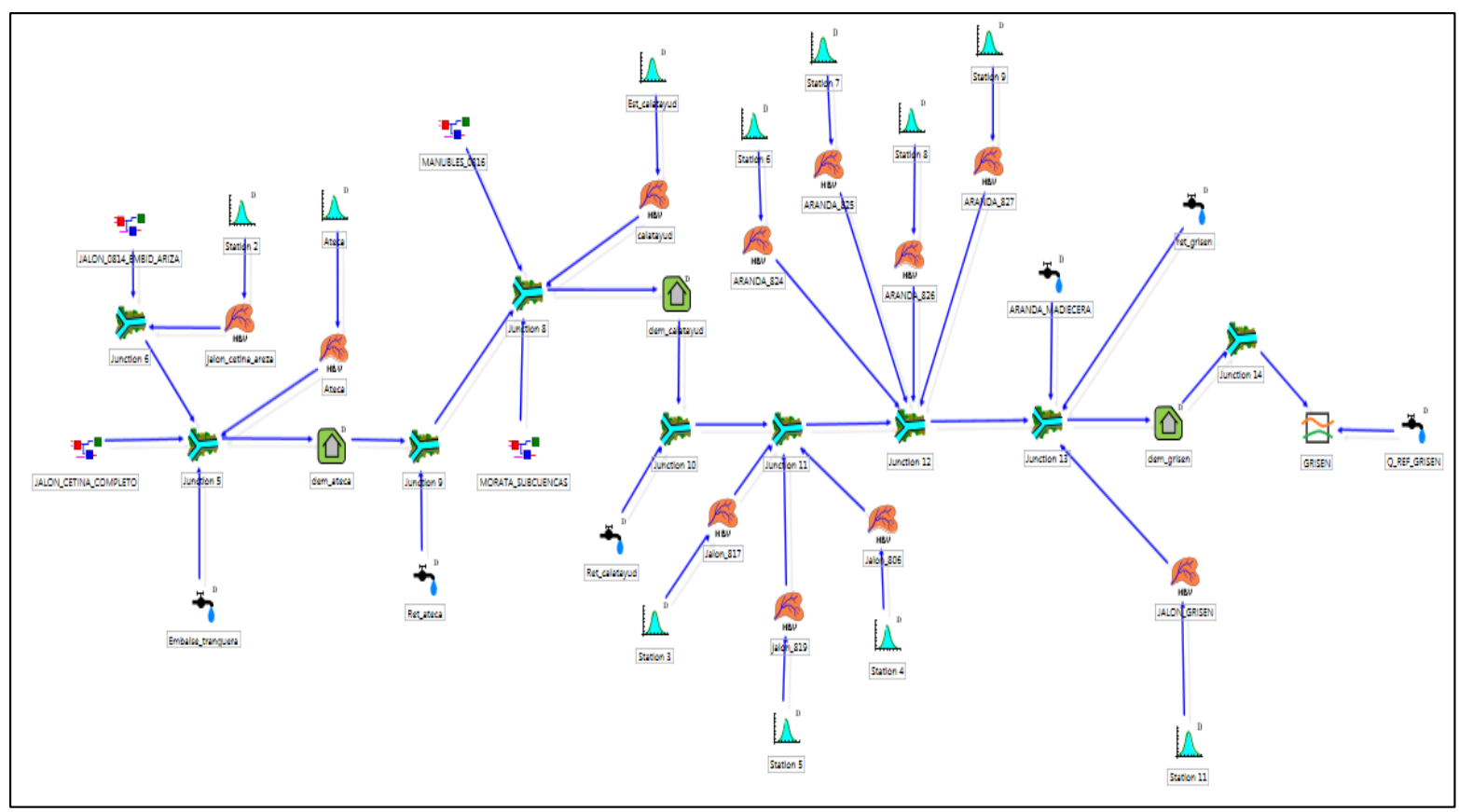


Tabla 1: Resumen de las Calibraciones y validaciones del modelo MPE para el sistema de explotación del río Jalón.

\begin{tabular}{|c|c|c|c|c|c|}
\hline E AFORO & UNIDAD & $\begin{array}{c}\text { PERÍODO } \\
\text { CALIBRACIÓN }\end{array}$ & FO & $\begin{array}{c}\text { PERÍODO } \\
\text { VALIDACIÓN }\end{array}$ & FO \\
\hline \multicolumn{6}{|c|}{ SISTEMA JILOCA } \\
\hline Pancrudo & 912 & $1986-2001$ & 0.8001 & $2001-2006$ & 0.7206 \\
\hline Daroca & 908 & 1986-2001 & 0.9351 & 2001-2006 & 0.8804 \\
\hline Morata & 909,911 & $1986-2001$ & 0.6408 & 2001-2006 & 0.7495 \\
\hline \multicolumn{6}{|c|}{ SISTEMA PIEDRA } \\
\hline Járaba & 905 & $1987-2001$ & 0.7539 & 2001-2006 & 0.6570 \\
\hline Nuévalos & 901,902 & 1984-1997 & 0.8293 & $1997-2000$ & 0.7005 \\
\hline Tranquera & 906 & $1987-2001$ & 0.7518 & $2001-2007$ & 0.7968 \\
\hline \multicolumn{6}{|c|}{ SISTEMA MANUBLES } \\
\hline $\begin{array}{l}\text { Ateca } \\
\text { SISTEMA }\end{array}$ & ARANDA & 1989-2000 & 0.7900 & $2000-2006$ & 0.5988 \\
\hline $\begin{array}{l}\text { Maidevera } \\
\text { SISTEMA }\end{array}$ & JALÓN & 1991-2002 & 0.7385 & $2002-2007$ & 0.7758 \\
\hline Barrio & 813 & $1996-2003$ & 0.7803 & 2003-2007 & 0.8184 \\
\hline Jubera & 801 & $1987-2001$ & 0.8479 & 2001-2007 & 0.8565 \\
\hline Ateca & 803 & $1987-2001$ & 0.777 & 2001-2007 & 0.8799 \\
\hline Calatayud & 804,91 & 1994-2001 & 0.8905 & $2001-2007$ & 0.8510 \\
\hline Chodes & $806,817,819$ & $2000-2005$ & 0.8554 & $2005-2008$ & 0.6675 \\
\hline Grisen & $\begin{array}{l}807,821,822,82 \\
4,825,827\end{array}$ & $1987-1998$ & 0.8309 & 2004-2007 & 0.6745 \\
\hline
\end{tabular}

Figura 4. a) Sistema de Explotación del Río Jalón, b) Calibración del río Jiloca, c) Calibración del río Piedra, d) Calibración del río Manubles, e) Calibración del río Isuelas, f) Calibración del sistema en conjunto del sistema de explotación del río Jalón
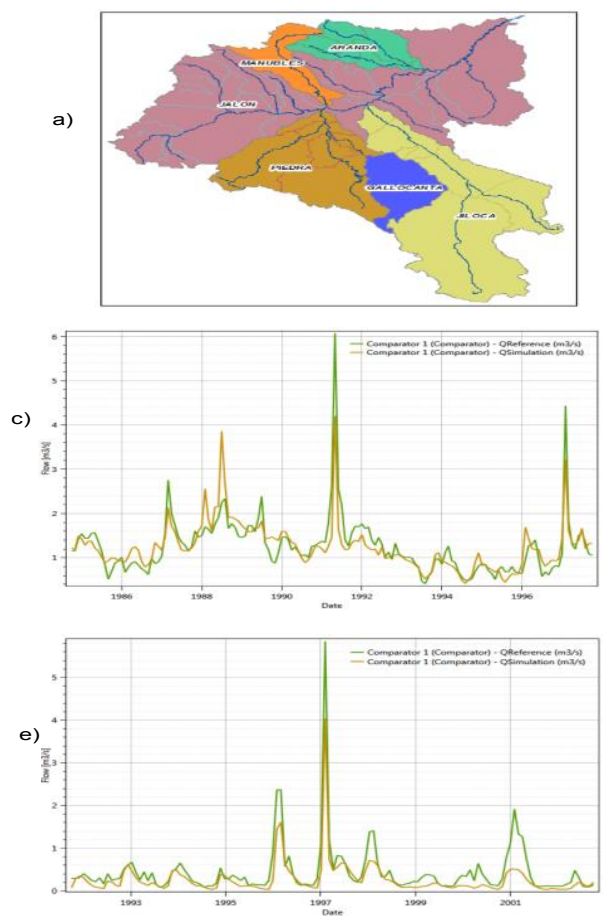

b)
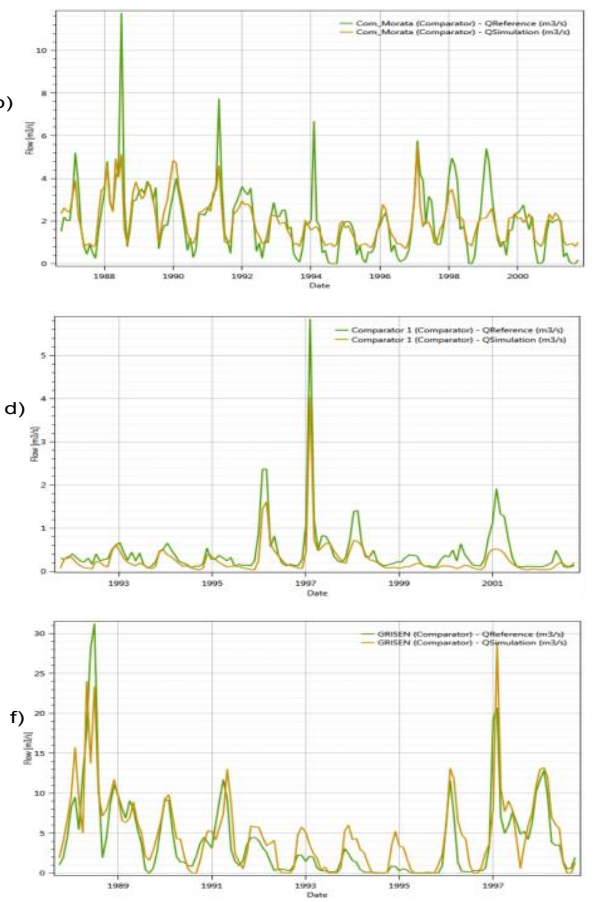


\section{Discusión}

Según el análisis de calibración del modelo de precipitación y escorrentía, y considerando los valores de la función objetivo (Figura 4), se deduce los mejores ajustes se obtuvieron sobre todo en aquellas subcuencas de cabecera, mismas que no abarcaban una gran extensión y que a la vez no se veían afectadas en gran medida por las detracciones de agua para satisfacer demandas ya sea de tipo agraria, urbana o industrial. Las cuencas de cabecera al encontrarse en régimen natural facilitaron y favorecieron el ajuste y calibración adecuada de los caudales simulados frente a los observados. Una vez conocido en cierto grado la naturaleza y el comportamiento de la zona mediante la aplicación del modelo HBV, a partir de las respuestas (aportaciones) obtenidas en cada sistema mediante el software Rs Minerve, se comparó con las aportaciones resultantes del modelo SIMPA (Simulación Precipitación- Aportación), desarrollado por el Centro de Estudios Hidrográficos del Centro de Estudios y Experimentación de Obras Públicas (CEDEX), que es el modelo que emplea la Confederación Hidrográfica del Ebro para la gestión y evaluación de los recursos hídricos en régimen natural para determinar las aportaciones del sistema de explotación del río Jalón.

Como se muestra en la Figura 5, las aportaciones del modelo SIMPA durante el periodo analizado (1950-2006) se encuentran casi en su totalidad por encima de las del modelo HBV, estas en varios casos como en el año hidrológico 1987 se encuentran sobreestimando los caudales circulantes en el sistema en gran proporción siendo junio/ del año hidrológico 1987 el punto de mayor diferencia entre los dos modelos $\left(91.47 \mathrm{Hm}^{3} / \mathrm{mes}\right)$. En toda la serie se evidencia que los picos de caudales distan en la mayoría de los casos aproximadamente $15 \mathrm{Hm} 3 / \mathrm{mes}$. Para el caso de la estimación de caudales bajos estos se correlacionan más entre sí, siendo en este caso que las aportaciones del modelo HBV se encuentran en todo momento en igualdad o ligeramente inferiores a las estimaciones realizadas por el modelo SIMPA.

Figura 5. Aportaciones anuales del Sistema de Explotación del río Jalón.

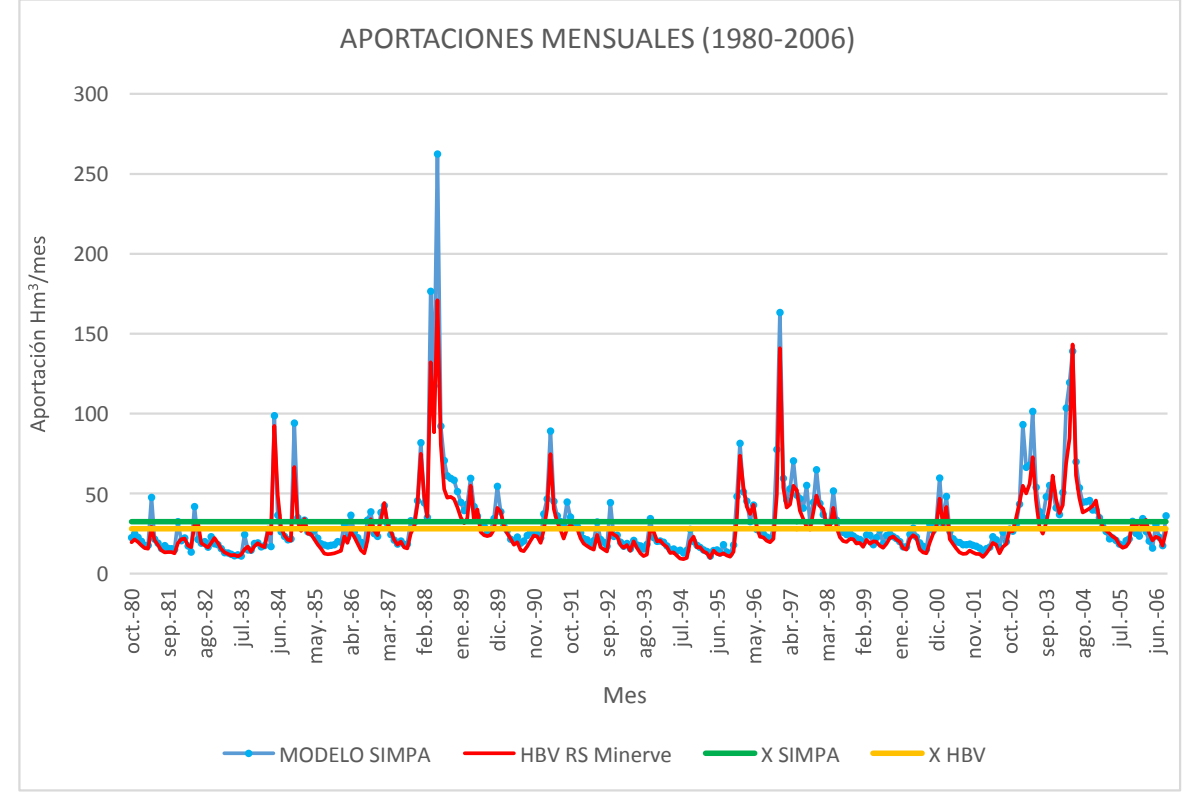




\section{Conclusiones}

- En este estudio se desarrolló la evaluación de los recursos hídricos del sistema del río Jalón a través de la aplicación de modelos de precipitación-escorrentía en forma semidistribuida empleando la herramienta Rs Minerve 2.0 la cual mediante el modelo HBV permitió analizar el comportamiento de los recursos hídricos, flujos superficiales, subterráneos e influencia que ejercen las estructuras de regulación en todas las subcuencas que forman parte del sistema de explotación.

- Los resultados del análisis en desembocadura calibrados y validados (0.83 y 0.67 respectivamente) del sistema de explotación del río Jalón fueron buenos a pesar de la incertidumbre que conllevan los MPE y de los errores sistemáticos de calibración mientras más aguas abajo nos encontramos en el sistema, esto debido a las alteraciones que se derivan de los embalses, y a las importantes detracciones de agua para satisfacer las demandas.

- Realizado el análisis se deduce que el modelo SIMPA se encuentra sobreestimando los caudales, mismas que pueden tener repercusiones al momento de la gestión de la demanda, dado que al hipotéticamente disponer de más recursos se podría asignar los mismos a otro tipo de demandas, sin embargo si en la realidad estas aportaciones estimadas no se cumplen se podría generar fallos en el sistema, razón por la cual se debe tener cuidado al momento de tomar decisiones, en la planificación y gestión de los recursos, teniendo en cuenta que siempre debemos estar del lado de la seguridad.

- Finalmente, hay que señalar que los modelos de precipitación constituyen una herramienta indispensable en la gestión de los recursos hídricos, las aportaciones generadas por los MPE generalmente se emplean como entradas a los modelos de gestión cuyo objetivo principal es la estimación de garantías de suministro a las demandas, predicción de avenidas, gestionar adecuadamente los recursos, entre otras. Además, es importante recordar que la efectividad del modelo y su capacidad de predicción dependen en gran parte de la forma y circunstancias bajo las cuales fueron elaboradas y principalmente de la comprensión y entendimiento de los procesos hidrológicos que se desarrollan en la cuenca que se está estudiando.

\section{Agradecimiento}

- A la Confederación Hidrográfica del rio Jalón, por ser el ente que facilitó el régimen de aportaciones para el dearrollo del modelo.

\section{Referencias Bibliograficas}

Abbott, M. B., Bathurst, J. C., Cunge, J. A., O'connell, P. E., \& Rasmussen, J. (1986b). An introduction to the European Hydrological System-Systeme Hydrologique Europeen, "SHE", 2: Structure of a physically-based, distributed modelling system. Journal of 
hydrology, 87(1), 61-77.

Bates, P. D., \& De Roo, A. P. J. (2000). A simple raster-based model for flood inundation simulation. Journal of hydrology, 236(1), 54-77.

Benoit, R., Pellerin, P., Kouwen, N., Ritchie, H., Donaldson, N., Joe, P., \& Soulis, E. D. (2000). Toward the use of coupled atmospheric and hydrologic models at regional scale. Monthly Weather Review, 128(6), 1681- 1706.

Bergström, S., \& Singh, V. P. (1995). The HBV model. Computer models of watershed hydrology. 443-476.

De Roo, A. P. J. (1998). Modelling runoff and sediment transport in catchments using GIS. Hydrological processes, 12(6), 905-922.

De vos, N.J., Rientjes, T.H.M. and Savenije, H.H.G, 2006 Enhanced conceptual rainfall runoff modeling through ensemble Kalman filtering.

Ewen, J., Parkin, G., \& O'Connell, P. E. (2000). SHETRAN: distributed river basin flow and transport modeling system. Journal of hydrologic engineering, 5(3), 250-258.

García Hernández, J., Boillat, J.-L., Feller, I. and Schleiss A. J. (2013). Présent et futur des prévisions hydrologiques pour la gestion des crues. Le cas du Rhône alpin. Mémoire de la Société vaudoise des Sciences naturelles 25: 55-70. ISSN 0037-9611.

Garcia-Bartual, R. (2002). Short term river flood forecasting with neural networks. Universidad Politecnica de Valencia, Spain, 160-165.

Hargreaves, G. H., \& Samani, Z. A. (1985). Reference crop evapotranspiration from temperature. Applied engineering in agriculture, 1(2), 96-99.

Herrera, S., Gutiérrez, J. M., Ancell, R., Pons, M. R., Frías, M. D., \& Fernández, J. (2012). Development and analysis of a 50-year high-resolution daily gridded precipitation dataset over Spain (Spain02). International Journal of Climatology, 32(1), 74-85

Jordan, F., Boillat, J.-L. And Schleiss, A. J. (2010). Prévision et gestion des crues du Rhône supérieur par l'exploitation optimale des retenues alpine. La Houille Blanche, No. 5, 91-102.

Lindström, G., Johansson, B., Persson, M., Gardelin, M., \& Bergström, S. (1997).

Development and test of the distributed HBV-96 hydrological model. Journal of hydrology, 201(1), 272-288.

Mulvany, W. T., Fraser, W., Roberts, S., Mulvany, T. J., \& O'Flaherty, J. (1850). Appendix No. V: Notices of Antiquities Presented to the Royal Irish Academy by WT Mulvany, Esq., MRIA, on the Part of the Commissioners of Public Works. Proceedings of the Royal Irish Academy (1836-1869), 5, xxxi-lxvi.

Refsgaard, J. C., Storm, B., \& Refsgaard, A. (1995). Recent developments of the Systeme Hydrologique Europeen(SHE) towards the MIKE SHE. International Association of Hydrological Sciences, Publication, (231), 427-434.

Sherman, L. K. (1932). Streamflow from rainfall by the unit-graph method. Eng. News 
Record, 108, 501-505.

Taffese, T. E. W. O. D. R. O. S. (2011). Physically based rainfall: Runoff modelling in the northern Ethiopian highlands: The case of Mizewa watershed.

Vivoni, E. R., \& Camilli, R. (2003). Real-time streaming of environmental field data. Computers \& Geosciences, 29(4), 457-468.

Wigmosta, M. S., Vail, L. W., \& Lettenmaier, D. P. (1994). A distributed hydrology-vegetation model for complex terrain. Water resources research, 30(6), 1665-1679.

\section{PARA CITAR EL ARTÍCULO INDEXADO.}

Chuquin, D., Chuquin, N., Chuquin, J., Castro, L., \& Cepeda, R. (2019). Desarrollo de un modelo de precipitación escorrentía semi-distribuido para la evaluación de los recursos hídricos del río Jalón. Ciencia Digital, 3(3.1), 115-126. https://doi.org/10.33262/cienciadigital.v3i3.1.680

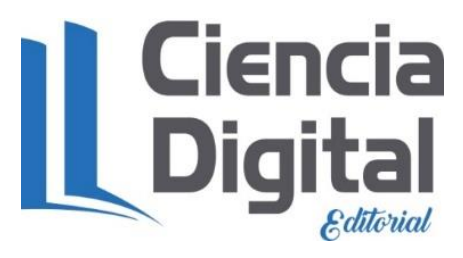

El artículo que se publica es de exclusiva responsabilidad de los autores y no necesariamente reflejan el pensamiento de la Revista Ciencia Digital.

El artículo queda en propiedad de la revista y, por tanto, su publicación parcial y/o total en otro medio tiene que ser autorizado por el director de la Revista Ciencia Digital.
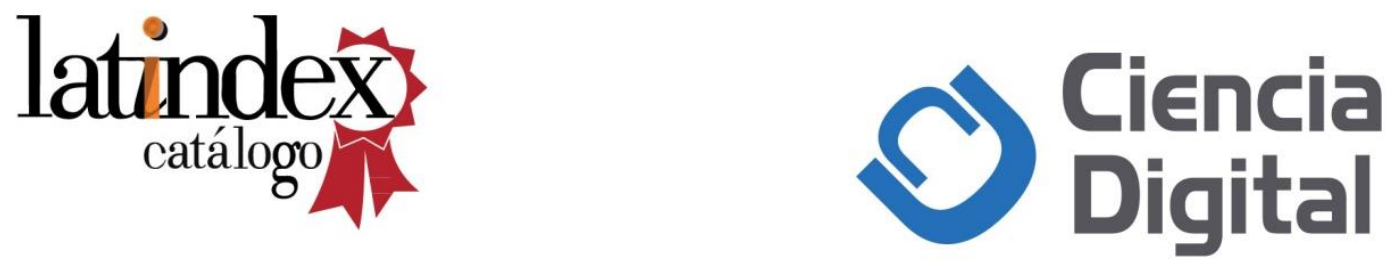